\title{
Modeling of Proton-Induced CCD Degradation in the Chandra X-Ray Observatory
}

\author{
D. H. Lo and J. R. Srour, Fellow, IEEE
}

\begin{abstract}
Modeling results are presented for proton-induced degradation of charge-coupled devices (CCDs) used in the Advanced CCD Imaging Spectrometer instrument on the Chandra X-Ray Observatory. A methodology is described that provides insights regarding degradation mechanisms and onorbit performance for front-illuminated and back-illuminated CCDs. Proton-induced changes in charge transfer inefficiency are modeled. The observed amount of on-orbit degradation can be accounted for using a proton spectrum at the CCD location that is reduced in magnitude by a factor of $-1 E 5$ compared to the spectrum incident on the spacecraft.
\end{abstract}

\section{INTRODUCTION AND BACKGROUND}

THIS paper presents results of modeling activities that 1 examined proton-induced degradation of charge-coupled devices used in the Advanced CCD Imaging Spectrometer (ACIS) instrument on the Chandra X-Ray Observatory, which was launched on July 23, 1999. ACIS consists of an imaging array that includes four front-illuminated (FI) CCDs and a grating (or spectrometer) array that includes six CCDs, four of which are front-illuminated and two of which are backilluminated (BI). Front-illuminated CCDs were observed to exhibit charge transfer inefficiency (CTI) degradation during the first month of ACIS operation. In contrast, backilluminated ACIS CCDs did not degrade. Several workers subsequently described the Chandra radiation environment, the observed CCD degradation, modeling activities, and mitigation approaches [1]-[7]. The general conclusion reached was that a fraction of the protons entering the $x$-ray telescope aperture are scattered to the CCD location and cause performance degradation. Related studies were conducted for the XMM-Newton X-ray Observatory [7] and the ASCA x-ray astronomy satellite [8].

A detailed modeling methodology is presented herein that offers insights regarding degradation mechanisms and onorbit performance for $\mathrm{BI}$ and FI devices used in the ACIS instrument. Proton-induced changes in charge transfer inefficiency are considered. The CTI modeling results are employed to provide an estimate for that fraction of the proton fluence incident on the spacecraft that reaches the CCDs. Our modeling of ACIS CCD degradation was

Manuscript received March 2003. This work was supported in part by NASA under Contract No. NAS 8-37710

The authors are with Northrop Grumman Space Technology, Redondo Beach, CA 90278 USA performed a few months after the Chandra launch [9]. The present work describes that modeling effort and then compares the results with those obtained by other workers.

\section{DEVICE STRucture}

Figures 1 and 2 present cross-sections for FI and BI CCDs, respectively, based on information compiled from several sources [10]-[13]. The OBF (Optical Blocking Filter) used in ACIS is also included in each cross-section since it provides some shielding of the CCDs to incident protons. The OBF dimensions shown are those for the filter used with the grating array of six CCDs. (For the four imaging-array CCDs, the thickness of the bottom $\mathrm{Al}$ layer in the OBF is $0.13 \mu \mathrm{m}$. Thicknesses of the other two layers in the OBF were the same for the grating and imaging arrays.) Figure 3 presents the doping profile for the n-layer in FI and BI devices [11]. This profile yields a junction depth of $\sim 0.48 \mu \mathrm{m}$ and a substrate doping density of $2.7 \mathrm{E} 12 \mathrm{~cm}^{-3}$, or $5000 \Omega-\mathrm{cm}$. Figure 4 shows the simulated potential in the n-layer under the assumption of no fixed oxide charge and for a gate voltage of $0 \mathrm{~V} \mathrm{[11].} \mathrm{This} \mathrm{simulation} \mathrm{provides} \mathrm{an} \mathrm{estimate} \mathrm{of} \mathrm{the} \mathrm{depth}$ into the n-layer of the middle of the buried channel $(\sim 0.32$ $\mu \mathrm{m}$ ). A buried channel (BC) width of $\sim 0.1 \mu \mathrm{m}$ is assumed.

\section{MODELING METHODOLOGY}

The proton energy spectrum incident on ACIS CCDs was uncertain when our modeling activities [9] were conducted. Work was performed subsequently to estimate that information [3], [7]. In general, the spectrum incident on the spacecraft will be altered during transit to the CCDs through the effects of shielding and scattering. The proton fluence will be reduced substantially at the CCD location compared to the fluence incident on the spacecraft. The simplifying assumption was made in our modeling that the shape of the proton energy spectrum incident on the OBF is the same as that incident on the spacecraft. (To simplify the analysis, normal incidence on the OBF and the CCDs is also assumed.) It is further assumed initially that the fluence at a given energy incident on the OBF is also the same as that striking the spacecraft. We then deduce both the fraction of the external proton fluence and the effective acceptance angle for incident protons that would produce the CCD degradation observed in space. 


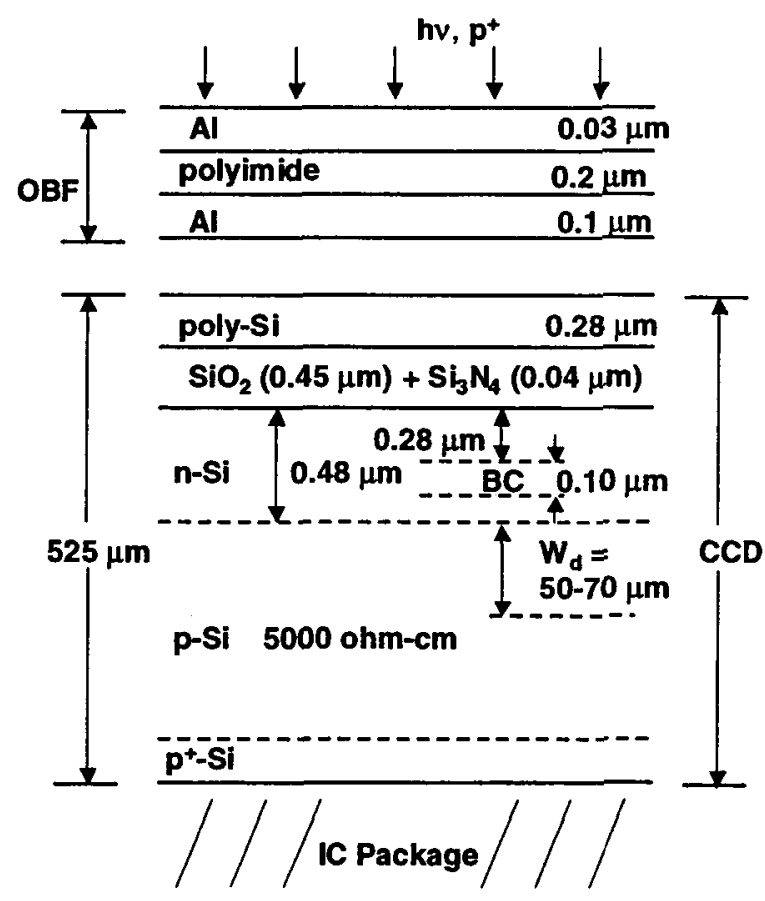

Fig. 1. Cross-section of FI CCD. The OBF dimensions are for the filter used with the ACIS grating array.

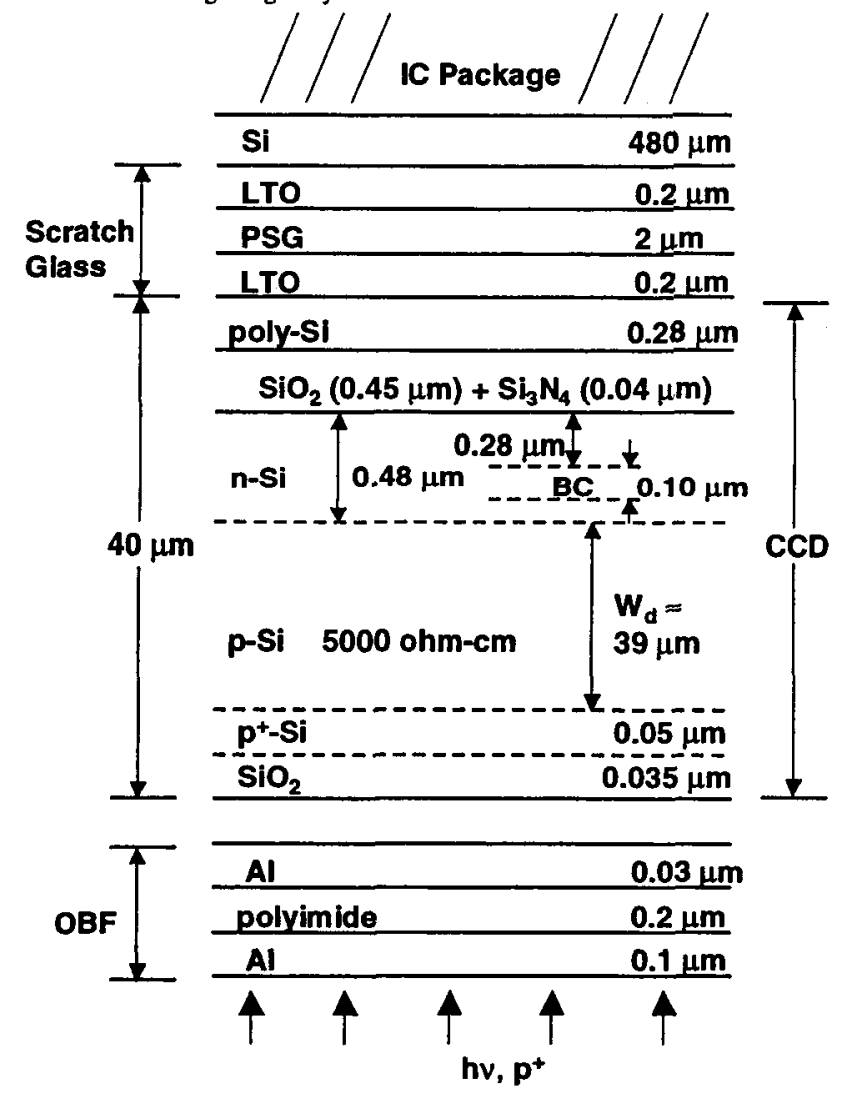

Fig. 2. Cross-section of $\mathrm{BI} \mathrm{CCD}$.
The modeling methodology involves transporting the proton spectrum through the filter and the intervening $C C D$ structure until protons, with degraded energy, reach the buried channel in FI and BI devices. The average dose deposited in nonionizing processes (i.e., the displacement damage dose, $\left.D_{d}\right)$ in the buried channel for both types of CCDs is then determined through detailed analyses. That dose is used in conjunction with space and ground measurements of CTI degradation to estimate that fraction of the proton fluence incident on the spacecraft that reaches the ACIS CCDs and the corresponding effective acceptance angle. Shielding effects of the HETG (High Energy Transmission Grating) used with ACIS are also considered.

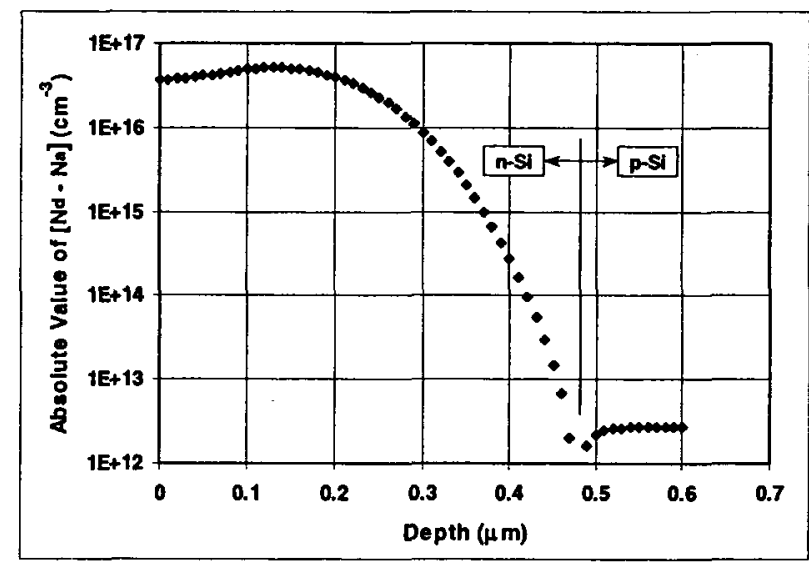

Fig. 3. Doping profile versus depth into the n-layer [11].

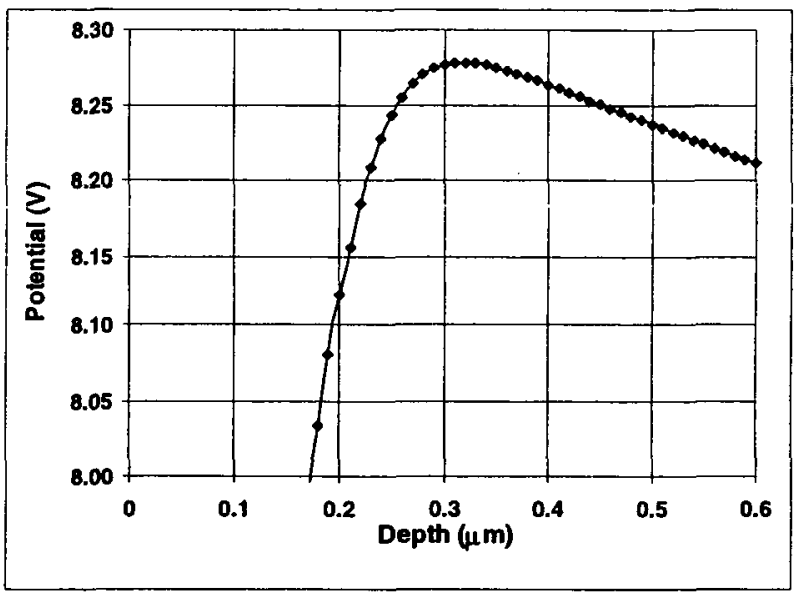

Fig. 4. Potential profile versus depth into the n-layer [11].

\section{PROTON SPECTRA}

Figure 5 shows the proton spectrum incident on the spacecraft plotted in terms of differential fluence in units of protons per $\mathrm{cm}^{2}$-steradian-orbit-MeV. This input spectrum was calculated using the Space Radiation code, which restricts the lower-energy limit to $100 \mathrm{keV}$. (The validity of the underlying AP8 model is questionable at lower energies.) 
Transport calculations require knowledge of the range-energy relation, $R(E)$, for protons incident on a silicon target. The SRIM code [14] was used to determine $R(E)$, which is shown in Figure 6. To simplify the modeling, it was then assumed that all protons lose their energy in a manner consistent with the range-energy relation (i.e., no straggling). Thus, for a proton with energy $E$ to reach a specific depth $x$ in silicon, the criterion $R(E)>x$ must be met. Upon reaching depth $x$, the proton will have an energy $E_{x}$ such that $R(E)=R\left(E_{x}\right)+x$. In this manner, the input proton energy spectrum can be transported to any depth of interest.

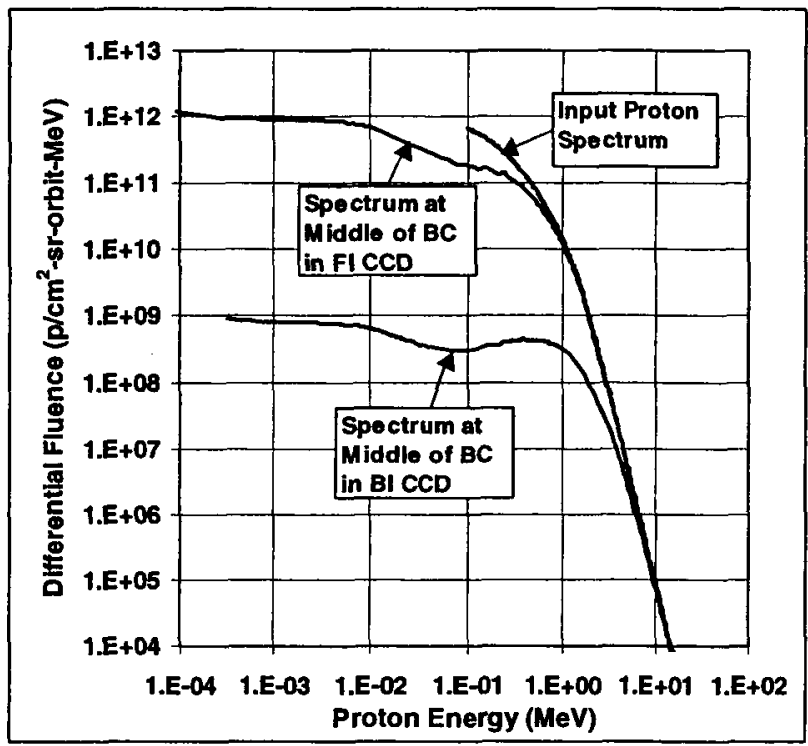

Figure 5. Proton differential fluence spectra. The spectrum incident on the spacecraft is shown as well as that spectrum transported to the middle of the buried channel for FI and BI CCDs. The simplifying initial assumption was made that the proton energy spectrum and fluences incident on the $O B F$ are the same as that incident on the spacecraft.

It is important to determine the transported proton energy spectrum for energies well below $100 \mathrm{keV}$ in device active regions. The nonionizing energy loss rate (NIEL), which correlates with the amount of displacement damage produced, increases with decreasing proton energy [15] (Figure 6), and thus low-energy protons contribute significantly to device degradation. This subject is discussed in [16], [17]. (Silicon NIEL values were available in the present study for proton energies of $1 \mathrm{keV}$ and higher. Our calculations of Si NIEL for lower energies (100 eV to $1 \mathrm{keV})$ indicate that displacement damage doses reported below for the buried channel may be underestimates by $\sim 10 \%$.) Figure 5 also shows the differential proton spectra for FI and BI devices at the center of the buried channel, i.e., after transporting the input spectrum to that location in both cases.

For FI CCDs, in our modeling approach $100-\mathrm{keV}$ protons do not reach the buried channel because their range in $\mathrm{Si}$ equivalent material is $\sim 0.92 \mu \mathrm{m}$, whereas the edge of the $\mathrm{BC}$ is at a Si-equivalent depth of $\sim 1.3 \mu \mathrm{m}$ (including the $\mathrm{OBF}$ and the top layers of the CCD). However, most of the protons with energy $>100 \mathrm{keV}$ reach the $\mathrm{BC}$ and deposit nonionizing dose therein. In the case of the BI CCDs, the distance required for a proton to reach the buried channel is tens of micrometers. Therefore, protons in the input spectrum with energy below $100 \mathrm{keV}$ do not matter in the analysis of CTI degradation. (Protons reaching the buried channel in both FI and $\mathrm{BI}$ devices with degraded energies $<100 \mathrm{keV}$ all have energies $>100 \mathrm{keV}$ in the incident spectrum.)

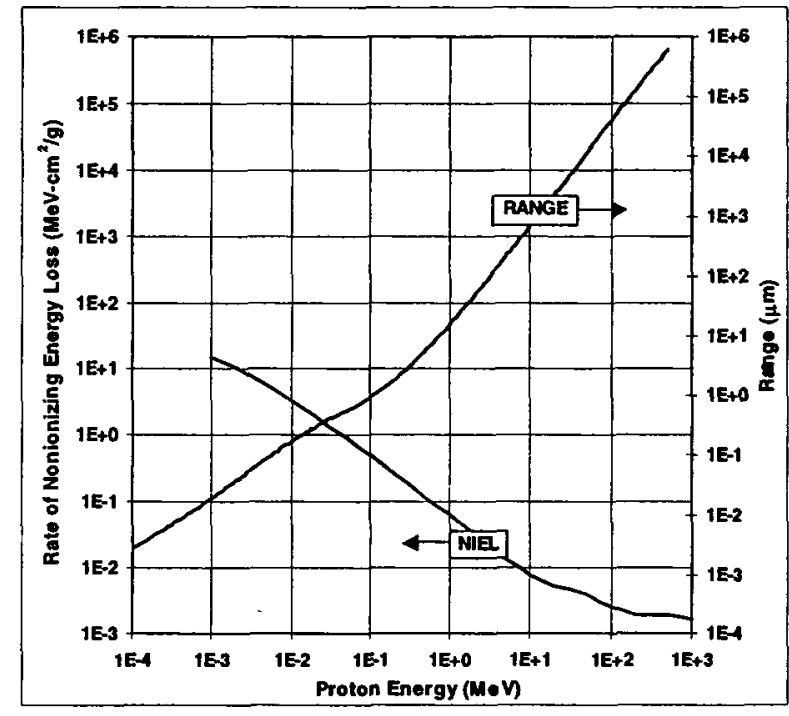

Fig. 6. Rate of nonionizing energy loss [15] and range versus incident proton energy for silicon.

\section{NONIONIZING ENERGY DEPOSITION}

The following approach was used to determine nonionizing energy deposition in CCD active regions. The input proton spectrum (Figure 5) was transported through the OBF and the various device layers (Figures 1 and 2 ) in the manner noted above. At each depth increment into the device, a proton energy spectrum was obtained, such as those shown in Figure 5 for the middle of the buried channels in FI and BI devices. At each of those depth increments, the displacement damage dose was calculated by integrating the product of the fluence at that depth and the NIEL over the entire energy spectrum. That process was repeated through the entire active region of the CCDs. Figure 7 shows displacement damage dose versus depth determined in that manner. The curve shown is the nonionizing energy deposition profile for silicon. To use this curve for the other materials in the OBF and the CCD, an equivalent thickness of silicon must be determined based on the density of a given material relative to $\mathrm{Si}$. The results presented in Figure 7 were used to obtain the nonionizing energy deposition profile in and near the buried channel. Figures 8 and 9 show the results for FI and BI CCDs, respectively. $D_{d}$ varies by $-10 \%$ over the buried channel width in the front-illuminated device, whereas for the BI CCD the variation is $<1 \%$. 


\section{ChaRge TRANSFer INEFFICIENCY DEgRADATION}

Dale et al. [18] demonstrated that changes in charge transfer efficiency (CTE) for proton-irradiated CCDs scale with NIEL. It seems reasonable to expect that the radiationinduced increase in CTI (termed $\triangle C T I$ ), where CTI = 1- CTE, in any buried-channel CCD will scale linearly with the displacement damage dose deposited in the device active region, i.e., in the buried channel. Nonionizing energy loss in that region produces traps that degrade the transfer efficiency. It is well established that there is a linear dependence of $\triangle C T I$ on irradiating particle fluence $(\phi)$ for both monoenergetic particles and a spectrum of particle energies. (For example, see [19], [20] and references therein.) Since $D_{d}$ is linearly proportional to $\phi$ for a specific particle type and energy, the linear dependence of $\Delta C T I$ on $D_{d}$ follows. (In general, to analyze the effects on CTI of a spectrum of irradiating particle energies, one must determine the contribution to $D_{d}$ in the device active region at each energy.) The constant of proportionality between $\Delta C T I$ and $D_{d}$ is expressed here by $\mathrm{K}_{\mathrm{CTI}}$ :

$\Delta C T I=K_{C T I} D_{d}$

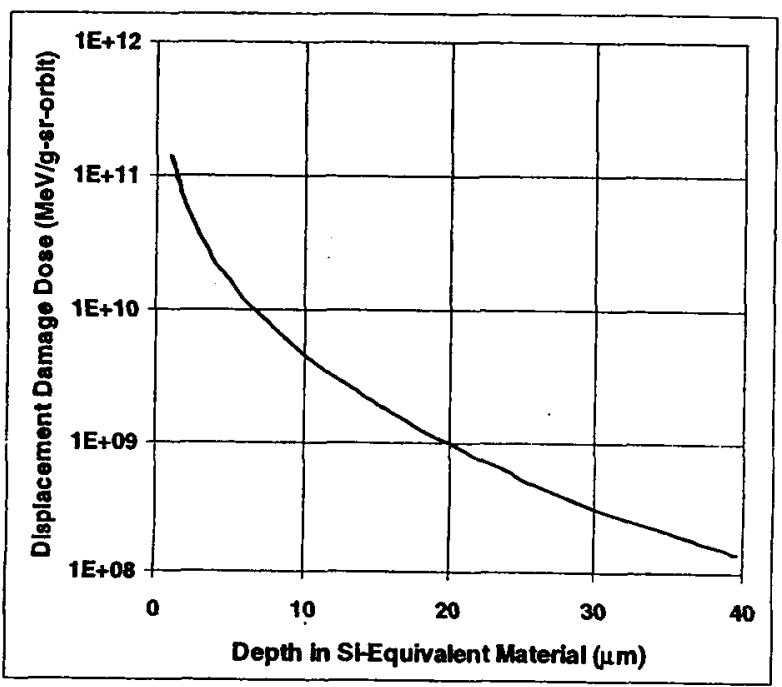

Fig. 7. Displacement damage dose deposited in FI and BI CCDs.

For the CCDs used on ACIS, ground measurements of CTI degradation following $40-\mathrm{MeV}$ proton irradiation were made prior to the Chandra launch. The value for $\mathrm{K}_{\mathrm{CT}}$ obtained in that work was expressed [21] as $(11 \pm 2)$ E-5 per nonionizing rad, where one nonionizing rad equals 6.25E7 MeV/g. Converting to the present units yields a value for $\mathrm{K}_{\mathrm{CrI}}$ of $(1.8$ $\pm 0.3) \mathrm{E}-12(\mathrm{MeV} / \mathrm{g})^{-1} .40-\mathrm{MeV}$ protons are quite penetrating and lose relatively little energy in traversing the $\mathrm{CCD}$. Thus, the NIEL value is well defined and equals that at $40 \mathrm{MeV}$ (i.e., 4.3E-3 $\mathrm{MeV}-\mathrm{cm}^{2} / \mathrm{g}$ ). Consequently, the displacement damage dose deposited in the device active region is known accurately, resulting in an accurate $\mathrm{K}_{\mathrm{CTI}}$ determination at that energy.

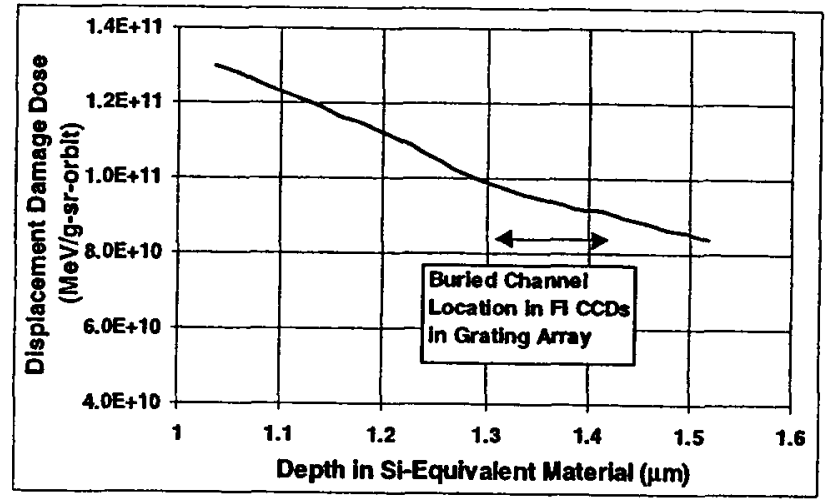

Fig.8. Displacement damage dose deposited in and near the buried channel in FI CCDs in the grating array on ACIS.

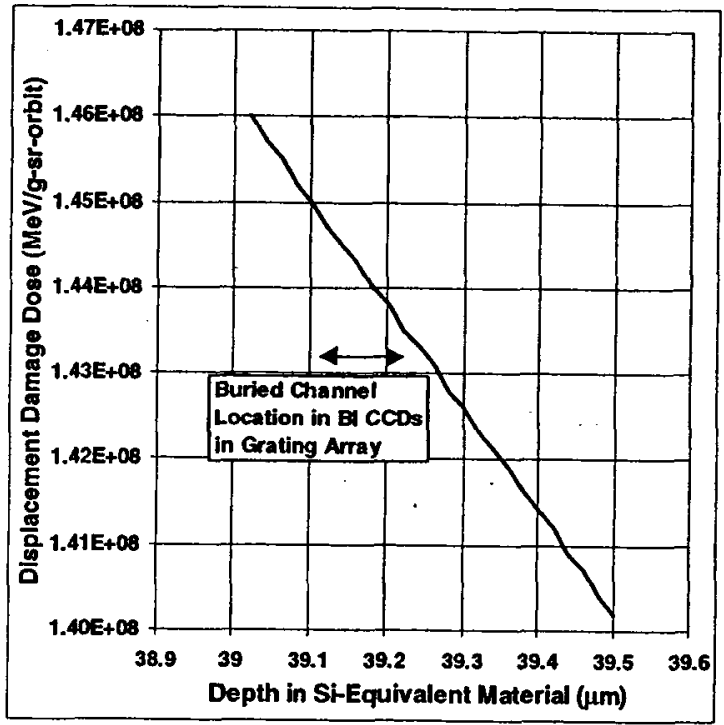

Fig. 9. Displacement damage dose deposited in and near the buried channel in BI CCDs in the grating array on ACIS.

Low-energy proton irradiations of ACIS CCDs at 104, 157, and $390 \mathrm{keV}$ were conducted at Goddard Space Flight Center using a van de Graaf accelerator [21]. The $K_{C T I}$ value obtained for 390-keV proton bombardment is $(8.4 \pm 0.6) \mathrm{E}-5$ per nonionizing rad [21], which converts to $(1.3 \pm 0.1) \mathrm{E}-12$ $(\mathrm{MeV} / \mathrm{g})^{-1}$. The present modeling approach was used in conjunction with additional information in [21] to reassess that value. This reassessment was performed because of the possibility of displacement damage dose uncertainty. An incident $390-\mathrm{keV}$ proton loses a significant portion of its energy before it reaches the buried channel region $(-20 \%)$. Accurate determination of $\mathrm{K}_{\mathrm{CT}}$ requires careful determination of the displacement damage dose deposited in the buried channel. The present work yields a NIEL value of -0.19 $\mathrm{MeV}-\mathrm{cm}^{2} / \mathrm{g}$ for degraded $390-\mathrm{keV}$ protons that reach the buried channel. The measured value for $\Delta C T I$ per unit fluence 
is $4.2 \mathrm{E}-13 \mathrm{~cm}^{2}$ at $390 \mathrm{keV}$ [21]. Using the presently determined displacement damage dose per unit fluence (i.e., NIEL) with that $\triangle C T I$ value yields a revised value for $K_{C T I}$ of $(2.2 \pm 0.2) \mathrm{E}-12(\mathrm{MeV} / \mathrm{g})^{-1}$ at that energy. This approach yields good agreement between the $\mathrm{K}_{\mathrm{CTI}}$ values for $40-\mathrm{MeV}$ and 390-keV proton bombardment of ACIS CCDs (i.e., 1.8E12 and $2.2 \mathrm{E}-12$, respectively). In the analysis that follows, we employ $\mathrm{K}_{\mathrm{CTI}}=2 \mathrm{E}-12(\mathrm{MeV} / \mathrm{g})^{-1}$ for all proton energies. In general, $\mathrm{K}_{\mathrm{CT}}$ depends on the CTI measurement conditions (e.g., background charge, clocking conditions, temperature). We assume here that this $\mathrm{K}_{\mathrm{Cn}}$ value is applicable for the onorbit measurement conditions for ACIS CCDs.

Applying the present modeling approach to determine $\mathrm{K}_{\mathrm{CTI}}$ at $157 \mathrm{keV}$ based on CTI measurements given in [21] yields a value on the order of $1 \mathrm{E}-12(\mathrm{MeV} / \mathrm{g})^{-1}$. We consider this value to be in reasonably good agreement with that obtained at $390 \mathrm{keV}$ and $40 \mathrm{MeV}$ in view of the significant analytical uncertainties in the $157-\mathrm{keV}$ case. In our analysis, a $157-\mathrm{keV}$ proton loses nearly all its energy $(\sim 90 \%)$ before reaching the buried channel, and exhibits significant further degradation as it traverses that channel. Thus, NIEL values in the buried channel and the resulting displacement damage dose deposited therein are very dependent on the device dimensions assumed and on other modeling assumptions (normal incidence, no straggling, no spectral variation, etc.).

Consequently, the error in the calculated $\mathrm{D}_{d}$, and thus in $\mathrm{K}_{\mathrm{CTI}}$, is relatively large for incident $157-\mathrm{keV}$ protons.

In the present one-dimensional analysis, based strictly on the range-energy relationship $104-\mathrm{keV}$ protons do not quite have enough energy to reach the buried channel in FI devices, even with the OBF removed, so no degradation is predicted. However, CTI degradation was observed at $104 \mathrm{keV}$ in ground irradiations [21] performed with no OBF in place. This discrepancy between modeling predictions and experimental results may be due to the various assumptions made here. Those assumptions are critical for proton energies where the particle range is comparable to the location of the device active region. To explore this apparent discrepancy, we performed Monte Carlo transport calculations for 104-keV protons using SRIM [14]. Those calculations demonstrated that when straggling is accounted for, very-low-energy protons do reach the buried channel and will cause CTI degradation. However, we were unable to quantify the displacement damage dose deposited because accurate NIEL values are not known for those energies. Another possible reason for the apparent discrepancy is proton energy variations for the irradiation source used. In the present analysis, we assumed that the incident protons used in ground experiments were monoenergetic. To achieve a low-energy proton beam using a van de Graaf, a scattering foil is often used to degrade a higher energy beam. If the input beam energy is large compared to the desired output energy, the spread in the output energy spectrum can be significant. This possible spread in proton energies incident on the $\mathrm{CCD}$ would need to be accounted for carefully in a modeling approach such as that described herein.

\section{RESULTS AND DISCUSSION}

When the ACIS CCDs were exposed to the space radiation environment for eight Chandra orbits, the pre-irradiation CTI of roughly 1E-6 increased to $-2 \mathrm{E}-4$ in FI devices [1], [13]. Using Eq. (1) and the $\mathrm{K}_{\mathrm{CTI}}$ value determined above of $2 \mathrm{E}-12$ $(\mathrm{MeV} / \mathrm{g})^{-1}$, a displacement damage dose of $-1 \mathrm{E} 8 \mathrm{MeV} / \mathrm{g}$ deposited in the buried channel would cause that amount of degradation. We now compare that value with the $D_{d}$ calculated herein for the FI CCD buried channel, which assumed that the proton spectrum and fluence incident on the spacecraft is also incident on the OBF and CCDs. That comparison then allows an estimate to be made of that fraction of the incident proton fluence that reaches the CCDs. From Figure 8, the calculated displacement damage dose at the middle of the FI CCD buried channel is $-9.4 \mathrm{E} 10 \mathrm{MeV} / \mathrm{g}$ sr-orbit. For eight orbits, a dose of $-7.5 \mathrm{E} 11 \mathrm{MeV} / \mathrm{g}-\mathrm{sr}$ is obtained. For the dose deposited in the $\mathrm{BC}$ to be $1 \mathrm{E} 8 \mathrm{MeV} / \mathrm{g}$, as determined above, then the effective solid angle that admits protons to the CCD region needs to be $-1.3 \mathrm{E}-4 \mathrm{sr}$. Under this interpretation, the fraction of the omnidirectional proton fluence that reaches the CCD is then $-(1.3 \mathrm{E}-4) / 4 \pi$, or $1 \mathrm{E}-5$. The proton spectrum is assumed to remain unchanged, but the fluence is reduced by that factor.

For BI devices, based on Figure 9 the displacement damage dose deposited in the buried channel during eight orbits is 1.1E9 MeV/g-sr, as compared to $\sim 7.5 \mathrm{E} 11 \mathrm{MeV} / \mathrm{g}$-sr for FI CCDs. Applying the solid acceptance angle calculated above

(1.3E-4 sr), we obtain an estimated $D_{d}$ for the BI buried channel of $\sim 1.5 \mathrm{E} 5 \mathrm{MeV} / \mathrm{g}$. From Eq. (1), that dose is predicted to increase the CTI by $3 E-7$, which is negligible. Many orbits $(-5000)$ would be needed to deposit sufficient dose to cause the CTI in BI CCDs to increase to 2E-4.

The effect of the HETG is now considered. After the FI CCDs were observed to experience a CTI increase to $-2 \mathrm{E}-4$, there were two additional orbits in which the HETG was inserted and the HRMA (High Resolution Mirror Assembly) covers were open. For this situation, the HETG provides additional shielding of the $\mathrm{CCD}$ beyond that already considered. Using the same methodology as employed above, we calculated the effects of that shielding on the displacement damage dose deposited in the buried channel of FI CCDs.

The result is a $D_{d}$ per orbit of $-9 E 6 \mathrm{MeV} / \mathrm{g}$. This is $9 \%$ of the dose needed to cause the CTI to increase to $2 \mathrm{E}-4$ (i.e., $9 \%$ of $1 E 8 \mathrm{MeV} / \mathrm{g}$ ). Thus, after an orbit with the HETG in place the CTI is predicted to increase by $1.8 \mathrm{E}-5$. The resulting total CTl, $2.2 \mathrm{E}-4$, most likely would be noted as a negligible change compared to the previous measurement of $2 \mathrm{E}-4$, in agreement with results given in [13]. (Note that proton scattering in the HETG will decrease the fractional CTI contribution to $<9 \%$.) 
Kolodziejczak et al. [3] also analyzed proton-induced degradation of the ACIS FI CCDs. The differential proton spectrum they used was based on AP8MAX, as compared to AP8MIN in the present study. (In the critical energy range between 100 and $500 \mathrm{keV}$, the AP8MIN fluence values we used are $-50 \%$ higher than those obtained using AP8MAX and employed in [3].) Kolodziejczak performed scattering analyses to determine the solid acceptance angle for incident protons to reach the ACIS CCDs. For the case of simply scaling the input proton spectrum (i.e., fluence-scaling only) to achieve the observed CTI degradation, they obtained an acceptance angle of $1.6 \mathrm{E}-4 \mathrm{sr}$, which agrees well with the value of $1.3 \mathrm{E}-4 \mathrm{sr}$ obtained here. That close numerical agreement may be somewhat fortuitous in view of the differences between the analytical methodologies employed and the input proton spectra. To first order, this agreement shows that one can account for ACIS CCD degradation based on the damage produced by incident protons that reach the focal plane through a very narrow energy-independent effective acceptance angle.

In the scattering analyses performed by Kolodziejczak et al. [3], they determined the acceptance angle as a function of proton energy. When that dependence is accounted for, their modeling underestimated the observed CTI degradation by a factor of 3 to 4 . That apparent discrepancy led them to suggest that other processes may be important, such as damage produced by heavier ions. They also note that such conclusions depend on the accuracy of their scattering analyses.

Nartallo et al. [7] also analyzed proton scattering for the Chandra x-ray telescope. For a fixed acceptance angle, over the proton energy range from $100 \mathrm{keV}$ to $1.5 \mathrm{MeV}$, they obtained $\sim 9 \mathrm{E}-6$ as that fraction of the incident proton fluence that reaches the CCDs. (The energy units on Figure 3b in [7] are evidently mislabeled as $\mathrm{keV}$ instead of MeV.) Their result agrees closely with the value of $-1 \mathrm{E}-5$ obtained herein. This agreement indicates that the present simplified modeling approach provides a physically reasonable description of ACIS CCD degradation.

\section{CONCLUDING REMARKS}

A methodology for determining proton-induced degradation of FI CCDs used in the ACIS instrument on Chandra is presented in this paper. Results of CTI analyses and modeling are described. The results are consistent with on-orbit observations of ACIS CCD degradation. In summary, charge transfer inefficiency degradation in CCDs used in the ACIS instrument on Chandra appears to be accounted for quite well quantitatively in terms of nonionizing energy deposition in the buried channel by relatively low energy protons.

\section{ACKNOWLEDGMENTS}

Information provided by M. W. Bautz, B. E. Burke, and J. A. Gregory was very helpful in the preparation of this paper.

\section{REFERENCES}

[1] S. L. O'Dell, M. W. Bautz, W. C. Blackwell, Y. M. Butt, R. A. Cameron, R. F. Elsner, M. S. Gussenhoven, J. J. Kolodziejczak, J. I. Minow, R. M. Suggs, D. A. Swartz, A. F. Tennant, S. N. Virani, and $K$ Warren, "Radiation environment of the Chandra $X$-ray Observatory," Proc. SPIE, vol. 4141, pp. 99-110, December 2000.

[2] W. C. Blackwell, Jr., J. I. Minow, S. L. O'Dell, R. M. Suggs, D. A Swartz, A. F. Tennant, S. N. Virani, and K. M. Warren, "Modeling the Chandra space environment," Proc. SPIE, vol. 4141, pp. 111-122, December 2000.

[3] J. J. Kolodziejczak, R. F. Eisner, R. A. Austin, and S. L. O'Dell, "Ion transmission to the focal plane of the Chandra X-ray Observatory," Proc. SPIE, vol. 4140, pp. 135-143, December 2000.

[4] G. Prigozhin, S. Kissel, M. Bautz, C. Grant, B. LaMarr, R. Foster, G. Ricker, and G. Garmire, "Radiation damage in the Chandra $x$-ray CCDs," Proc. SPIE, vol. 4012, pp. 720-730, July 2000.

[5] G. Prigozhin, S. Kissel, M. Bautz, C. Grant, B. LaMarr, R. Foster, and G. Ricker, "Characterization of the radiation damage in the Chandra $x$ ray CCDs," Proc. SPIE, vol. 4140, pp. 123-134, December 2000.

[6] J. E. Hill, K. T. Lewis, R. F. Foster, M. W. Bautz, S. K. Brown, P. W A. Roming, A. E. Homschemeier, D. N. Burrows, and G. P. Garmire, "A simulation of the flight experience of the ACIS CCDs on the Chandra X-ray Observatory," Proc. SPIE, vol. 4012, pp, 402-413, July 2000.

[7] R. Nartallo, E. Daly, H. Evans, P. Nieminen, F. Lie, and P. Truscott, "Low-angle scattering of protons on the XMM-Newton optics and effects on the on-board CCD detectors," IEEE Trans. Nucl. Sci., vol. 48, no. 6, pp. 1815-1821, December 2001.

[8] A. Yamashita, T. Dotani, M. Bautz, G. Crew, H. Ezuka, K. Gendreau, T. Kotani, K. Mitsuda, C. Otani, A. Rasmussen, G. Ricker, and H. Tsunemi, "Radiation damage to charge coupled devices in the space environment," IEEE Trans. Nucl. Sci., vol. 44, no. 3, pp. 847-853, June 1997.

[9] J. R. Srour and D. H. Lo, "Modeling of Proton-lnduced Degradation in ACIS CCDs," TRW Technical Note, January 14, 2000.

[10] B. E. Burke, J. A. Gregory, M. W. Bautz, G. Y. Prigozhin, S. E. Kissel, B. B. Kosicki, A. H. Loomis, and D. Y. Young, "Soft-X-ray CCD imagers for AXAF," IEEE Trans. Electron Devices, vol. 44, no. 10, pp. 1633-1642, October 1997.

[11] B. E. Burke, personal communication, October 1999.

[12] J. A. Gregory, personal communication, October 1999.

[13] G. P. Garmire and J. A. Nousek, "Preliminary Status Report: ACIS Front-Illuminated CCD Detector Anomaly," Pennsylvania State University Report, October 6, 1999.

[14] J. F. Ziegler, SRIM (Stopping and Range of lons in Matter) Code, available online at http:/www.srim.org.

[15] G. P. Summers, E. A. Burke, P. Shapiro, S. R. Messenger, and R. J. Walters, "Damage correlations in semiconductors exposed to garnma, electron and proton irradiations," IEEE Trans. Nucl. Sci., vol. 40, no. 6, pp. 1372-1379, December 1993.

[16] S. R. Messenger, M. A. Xapsos, E. A. Burke, R. J. Walters, and G. P. Summers, "Proton displacement damage and ionizing dose for shielded devices in space," IEEE Trans. Nucl. Sci., vol. 44, no. 6, pp. 2169 2173, December 1997.

[17] S. R. Messenger, E. A. Burke, G. P. Summers, and R. J. Walters, "Application of displacement damage dose analysis to low-energy protons on silicon devices," IEEE Trans. Nucl. Sci., vol. 49, no. 6, pp. 2690-2694, December 2002.

[18] C. Dale, P. Marshall, B. Cummings, L. Shamey, and A. Holland, "Displacement damage effects in mixed particle environments for shielded spacecraft CCDs," IEEE Trans. Nucl. Sci., vol. 40, no. 6, pp. 1628-1637, December 1993.

[19] J. R. Srour, R. A. Hartmann, and S. Othmer, "Transient and permanent effects of neutron bombardment on a commercially available n-buriedchannel CCD," IEEE Trans. Nucl. Sci., vol. 27, no. 6, pp. 1402-1410, December 1980.

[20] G. R. Hopkinson, C. R. Dale, and P. W. Marshall, "Proton effects in charge-coupled devices," IEEE Trans. Nucl. Sci., vol. 43, no. 2, pp. 614-627, April 1996.

[21] M. W. Bautz, "Summary of Recent Low-Energy Proton Experiments with ACIS CCDs," MIT Status Report, October 29, 1999. 